\title{
Caracterização espectral de Paspalum notatum em diferentes níveis de adubação nitrogenada ${ }^{(1)}$
}

\author{
Eliana Lima da Fonseca ${ }^{(2)}$, Luís Mauro Gonçalves $\operatorname{Rosa}^{(3)}$ e Denise Cybis Fontana ${ }^{(3)}$
}

\begin{abstract}
Resumo - As propriedades espectrais da vegetação podem ser utilizadas para o monitoramento das condições de crescimento da vegetação ao longo do ano, assim como a estimativa da produtividade das espécies, por meio de técnicas de sensoriamento remoto. Para tanto, é necessário, primeiramente, caracterizar espectralmente as espécies que compõem a vegetação de um ecossistema. O objetivo deste trabalho foi caracterizar a reflectância do Paspalum notatum Flügge var. notatum crescendo em diferentes níveis de adubação nitrogenada. Foi realizado um experimento de campo na Estação Experimental Agronômica da Universidade Federal do Rio Grande do Sul (305'22" S e 5139'8" W), utilizando-se um espectrorradiômetro portátil, em parcelas de P. notatum adubadas com 0, $200 \mathrm{e}$ $400 \mathrm{~kg} \mathrm{ha}^{-1}$ de N. A resposta espectral do $P$. notatum foi alterada pelas variações na quantidade de biomassa do dossel, provocadas por diferenças nos níveis de adubação nitrogenada. Os aumentos na quantidade de biomassa provocaram diminuição da reflectância na porção visível do espectro e aumento na porção infravermelho, o que mostra que a análise da reflectância é um bom indicador das mudanças que ocorrem em um dossel.
\end{abstract}

Termos para indexação: biomassa, cobertura vegetal, reflectância, espectrometria.

\section{Spectral characterization of Paspalum notatum under different nitrogen fertilization}

\begin{abstract}
Remote sensing techniques using spectral properties of canopies may be used to monitor vegetation growth conditions throughout the year, as well as to estimate the yield of different species. However, before these techniques can be used, it is necessary to establish the spectral properties of each of the different species which make up the landscape. The objective of this study was to characterize the spectral reflectance of Paspalum notatum Flügge var. notatum, growing on different levels of nitrogen fertilization. A field experiment was carried out at the Agronomic Experimental Station of Universidade Federal do Rio Grande do Sul, Brazil (30'5'22" S, 51 $39^{\circ} 8^{\prime \prime}$ W), using a portable spectroradiometer, on plots with $P$. notatum fertilized with 0,200 and $400 \mathrm{~kg} \mathrm{ha}^{-1}$ of nitrogen. The spectral response of $P$. notatum changed in response to increases in biomass caused by the different levels of nitrogen fertilization used. Increases in biomass resulted in a reduction in reflectance on the visible portion of the spectrum and an increase on the infrared portion, indicating, therefore, that the reflectance analysis is a good indicator of plant canopy status.
\end{abstract}

Index terms: biomass, canopy, reflectance, spectrometry.

(1) Aceito para publicação em 5 de setembro de 2001 .

Extraído da Dissertação de Mestrado apresentada pelo primeiro autor à Universidade Federal do Rio Grande do Sul (UFRGS), Faculdade de Agronomia, Porto Alegre, RS.

(2) Embrapa-Centro de Pesquisa Agropecuária dos Tabuleiros Costeiros, Av. Beira Mar, 3.250, Caixa Postal 44, CEP 49025-040 Aracaju, SE. E-mail: eliana@cpatc.embrapa.br

(3) UFRGS, Fac. de Agronomia, Dep. de Plantas Forrageiras e Agrometeorologia, Caixa Postal 776, CEP 91501-970 Porto Alegre, RS. E-mail: Imrosa@vortex.ufrgs.br, dfontana@vortex.ufrgs.br

\section{Introdução}

A caracterização espectral de um alvo, também conhecida como assinatura espectral, é a representação gráfica da reflectância em faixas de comprimento de onda bem estreitas e adjacentes. Ela representa de forma detalhada o resultado da interação da energia radiante com o objeto em estudo. As variações de amplitude na caracterização espectral são indicadoras das propriedades espectrais dos objetos (Steffen \& Moraes, 1993). 
De modo geral, a reflectância da vegetação varia com o comprimento de onda. Na região do visível, a reflectância não ultrapassa $10 \%$ da radiação incidente e caracteriza-se pela absorção acentuada, produzida pelos pigmentos presentes nos cloroplastos das folhas (Steffen \& Moraes, 1993; Fontana, 1995). Os pigmentos que mais influenciam a reflectância, nesta faixa de comprimento de onda, são as clorofilas a e b, com dois picos de absorção, o maior nos comprimentos de onda de 649 e $665 \mathrm{~nm}$, e o menor, nos comprimentos de onda de 420 e $435 \mathrm{~nm}$, respectivamente, e os carotenóides com pico de absorção nos $480 \mathrm{~nm}$ (Hall \& Rao, 1994).

A partir de $700 \mathrm{~nm}$, nota-se um gradiente acentuado que marca a transição para uma elevada reflectância no infravermelho próximo, a qual corresponde a, aproximadamente, 40 a $60 \%$ da radiação incidente, mantendo-se alta até cerca de $1.300 \mathrm{~nm}$. A elevação na reflectância nesta faixa é conseqüência do espalhamento de radiação no mesófilo, que ocorre por força da interação entre a radiação e a estrutura interna das folhas, e, também, em virtude do maior número de folhas presentes no dossel (Patel et al., 1985; Walter-Shea et al., 1991; Law \& Waring, 1994).

A reflectância do dossel nas faixas de comprimento de onda do visível e do infravermelho próximo tem sido amplamente utilizada para descrever a arquitetura das plantas (tais como o índice de área foliar) como também sua biomassa (Scurlock \& Prince, 1993; Fernández et al., 1994).

Diferenças nos níveis de $\mathrm{N}$ nas plantas podem ser detectadas por meio de sensores remotos. O N influencia tanto a taxa de expansão quanto a divisão celular, determinando, assim, o tamanho final das folhas, o que faz com que o $\mathrm{N}$ seja um dos fatores determinantes da taxa de acúmulo de biomassa (Jensen et al., 1990). Um acréscimo no suprimento de $\mathrm{N}$ estimula o crescimento, atrasa a senescência, e muda a morfologia das plantas. Além disso, o aumento nos níveis de adubação nitrogenada causa um aumento significativo no conteúdo de clorofila das folhas (Fernández et al., 1994).

À medida que aumentam os níveis de $\mathrm{N}$ aplicados na adubação, observa-se diminuição na reflectância em todos os comprimentos de onda da porção visível do espectro, pelo aumento da quantidade de fo- lhas e cloroplastos para interagir com a radiação (Jensen et al., 1990; Fernández et al., 1994).

Em virtude das mudanças que ocorrem na reflectância do dossel, e em razão da quantidade de $\mathrm{N}$ disponível, a biomassa pode ser monitorada por meio de técnicas de sensoriamento remoto, sendo necessário, para tanto, um estudo detalhado da interação entre N, biomassa e reflectância em nível terrestre.

Este trabalho teve por objetivo fazer a caracterização espectral de Paspalum notatum submetido a diferentes níveis de adubação nitrogenada.

\section{Material e Métodos}

O experimento foi realizado na Estação Experimental Agronômica da Faculdade de Agronomia da Universidade Federal do Rio Grande do Sul, no Município de Eldorado do Sul (30'5'22" S, 51 $339^{\prime} 8^{\prime \prime} \mathrm{W}$, altitude $46 \mathrm{~m}$ ).

O clima da região, segundo a classificação de Köppen, é subtropical úmido, de verão quente, do tipo Cfa. A precipitação pluvial média anual é de $1.400 \mathrm{~mm}$. A radiação solar global é máxima no mês de dezembro, com média diária de $26,0 \mathrm{MJ} \mathrm{m}^{-2} \mathrm{dia}^{-1}$, e mínima no mês de junho, com média diária de $8,38 \mathrm{MJ} \mathrm{m}^{-2} \mathrm{dia}^{-1}$. Janeiro e fevereiro são os meses mais quentes, enquanto junho e julho são os meses mais frios (Bergamaschi \& Guadagnin, 1990). O solo da área experimental, de acordo com o Sistema Brasileiro de Classificação de Solos, é um Argissolo Vermelho (Embrapa, 1999).

A cultura utilizada foi o Paspalum notatum Flügge var. notatum, ecótipo André da Rocha (grama forquilha), implantada em janeiro de 1995, plantada em três canteiros de $4 \mathrm{~m}$ por $12 \mathrm{~m}$. A adubação, realizada em 16 de novembro de 1998, constou da aplicação em toda a área experimental de $60 \mathrm{~kg} \mathrm{ha}^{-1}$ de $\mathrm{P}_{2} \mathrm{O}_{5}$ e $60 \mathrm{~kg} \mathrm{ha}^{-1}$ de $\mathrm{K}_{2} \mathrm{O}$. Os tratamentos constituíram-se de 0,200 e $400 \mathrm{~kg} \mathrm{ha}^{-1}$ de $\mathrm{N}$ (sulfato de amônio). As doses de $\mathrm{N}$ foram aplicadas em uma única aplicação.

A área experimental foi cortada com roçadeira tratorizada, para uniformização das parcelas. Durante o curso do experimento, foram feitos mais dois cortes, para diminuir a quantidade de biomassa nos canteiros, um aos 58 (13/jan./1999) e, o outro, aos 84 (8/fev./1999) dias após o início do experimento (16/nov./1998). As parcelas foram irrigadas por aspersão durante todo o período experimental. As medições da reflectância do dossel foram feitas aos 4 (20/nov./1998), 11 (27/nov./1998), 33 (19/dez./1998), 44 (30/dez./1998), 60 (15/jan./1999), 66 (21/jan./1999) e 87 (11/fev./1999) dias após o início do experimento, nas diferentes doses de $\mathrm{N}$ aplicadas. 
A reflectância foi determinada pela razão entre a radiância do dossel e a radiância de uma superfície lambertiana ideal, utilizada como referência, por meio de um espectrorradiômetro, marca GER modelo MARK 5, com uma resolução espectral de $3 \mathrm{~nm}$, nos comprimentos de onda compreendidos entre $350 \mathrm{~nm}$ e $1.100 \mathrm{~nm}$. O espectrorradiômetro foi instalado em um tripé, de forma que as medições foram tomadas a $2 \mathrm{~m}$ acima do topo da cultura. Esta disposição permite um campo de visada de, aproximadamente, $0,03 \mathrm{~m}^{2}(0,14 \times 0,21 \mathrm{~m})$. Utilizou-se como referência uma placa plana, cujo espectro de reflectância é conhecido, marca Labsphere modelo SRT-50-050.

As medições da reflectância foram realizadas de modo que a cada leitura eram medidas a quantidade de energia refletida pelo dossel e pela placa de referência, simultaneamente. Este tipo de medição, própria para trabalhos de campo, considera o efeito da atmosfera igual nas duas medições (do dossel e da referência), minimizando esta causa de variação. As três medições da reflectância, em cada um dos três tratamentos, foram feitas entre as 11 e as 13 horas, ou seja, com o ângulo de elevação solar superior a $75^{\circ}$, visando à redução do efeito do ângulo solar na reflectância da vegetação.

Nas medições de matéria seca total, matéria seca verde (somente folhas) e índice de área foliar, foram coletadas amostras de plantas em uma área de $0,03 \mathrm{~m}^{2}(15 \times 20 \mathrm{~cm})$ rente ao solo, com três repetições para cada tratamento. O material vegetal coletado foi separado em material morto, colmo e folhas. O material morto foi desprezado, e os demais materiais foram secados em estufa com ar forçado, a $60^{\circ} \mathrm{C}$, até atingir massa constante, obtendo-se, assim, matéria seca verde e matéria seca de colmo. A matéria seca total foi obtida somando-se as duas primeiras.

Parte do material de folhas da amostra foi utilizada para calcular a área foliar específica. Foram medidos, aproximadamente, $100 \mathrm{~cm}^{2}$ de área foliar com um planímetro ótico eletrônico, marca Li-cor, modelo LI-3100+1, e as folhas medidas foram secadas separadamente das demais, até atingirem massa constante em estufa com ar forçado a $60^{\circ} \mathrm{C}$. A área foliar obtida foi dividida pela matéria seca, obtendo-se a área foliar específica.

$\mathrm{O}$ índice de área foliar foi obtido multiplicando-se a área foliar específica pela massa da matéria seca verde, e dividindo-se pela área de terreno amostrado. A análise estatística foi realizada aplicando-se a técnica de modelos lineares (SAS GLM); quando existiram diferenças significativas, as médias foram comparadas pelo teste de Tukey a 5\% de probabilidade (SAS Institute, 1988).

\section{Resultados e Discussão}

A testemunha ( $\left.0 \mathrm{~kg} \mathrm{ha}^{-1} \mathrm{de} \mathrm{N}\right)$ apresentou a menor quantidade de matéria seca (total e verde), em comparação com os demais tratamentos (Tabela 1). Em cada data de medição, a maior quantidade e uma quantidade intermediária de matéria seca foram obtidas, respectivamente, nos tratamentos que receberam 400 e $200 \mathrm{~kg} \mathrm{ha}^{-1}$ de N, mostrando existir associação entre os níveis de $\mathrm{N}$ e a produção de biomassa do dossel, conforme Marschner (1990).

Dentro dos tratamentos 200 e $400 \mathrm{~kg} \mathrm{ha}^{-1}$ de N na porção visível do espectro (370 a $700 \mathrm{~nm}$ ), os menores valores de reflectância encontraram-se associados aos maiores valores de matéria seca (total e verde) e de índice de área foliar (Tabela 1). Esta resposta devese, provavelmente, à maior quantidade de pigmentos presentes, principalmente às clorofilas e aos carotenos, em conseqüência da maior área foliar (maior número de folhas), determinando uma maior absorção da radiação incidente, diminuindo, assim, a fração refletida pela vegetação (Steffen \& Moraes, 1993; Fontana, 1995). Na faixa de comprimento de onda entre 700 e $950 \mathrm{~nm}$ (porção infravermelho do espectro), a resposta observada foi oposta à da porção visível do espectro. Nessa faixa, associados aos maiores valores de matéria seca (total e verde) e de índice de área foliar medidos, encontram-se os maiores valores de reflectância, nos tratamentos que receberam 200 e $400 \mathrm{~kg} \mathrm{ha}^{-1}$ de nitrogênio. O aumento na quantidade de biomassa provoca um aumento no espalhamento da radiação. Por causa do maior número de camadas de folhas presentes, ocorre o fenômeno denominado reflectância aditiva, o que provoca um aumento da reflectância total na porção infravermelho do espectro (Steffen \& Moraes, 1993; Fontana, 1995).

Os resultados do presente trabalho, tanto na porção do visível quanto na porção do infravermelho do espectro, foram semelhantes aos encontrados com relação a Lepedium sativum L., arroz (Oryza sativa), cevada (Hordeum vulgare L.), soja (Glycine max Merrill.), milho (Zea mays L.) e amendoim (Arachis hypogaea L.) (Curran \& Milton,1983; Patel et al., 1985; Jensen et al., 1990; Walter-Shea et al., 1991; Peñuelas et al., 1997; Fideles Filho et al., 1999).

No tratamento testemunha, tanto na porção visível como na porção infravermelho do espectro, não foi observada a tendência esperada para a relação entre a reflectância e a produção de biomassa (Tabela 1). Provavelmente, esta resposta foi decor- 
rente da desuniformidade do dossel, sujeito à heterogeneidade natural da fertilidade do solo. Dada a necessidade de acompanhamento temporal da evolução do dossel, os pontos de medição da reflectância foram mantidos os mesmos durante todo o experimento, e isto fez com que as coletas de biomassa fossem necessariamente realizadas em outro local dentro do canteiro. Como as medidas de reflectância e de biomassa não foram realizadas nos mesmos pontos dentro das parcelas, pode ter havido superestimação de uma ou de outra variável, resultando em uma resposta fora dos padrões esperados.

As curvas espectrais, nos diferentes tratamentos e dias de medição, mostram o padrão característico da vegetação verde, e apresentam baixa reflectância na região do visível, com um pico próximo aos $550 \mathrm{~nm}$; e um substancial incremento na reflectância, próximo a $730 \mathrm{~nm}$, a qual se mantém alta em toda a região do infravermelho medida (Figura 1). Na porção visível os valores da reflectância em todos os comprimentos de onda são mais altos no tratamento teste- munha e diminuem à medida que a produção de biomassa aumenta (Figura 1 e Tabela 1). A produção de biomassa foi semelhante tanto para o tratamento que proporcionou as menores reflectâncias (400 kg ha-1 de N) como para aquele que apresentou valores intermediários de reflectância $\left(200 \mathrm{~kg} \mathrm{ha}^{-1} \mathrm{de}\right.$ $\mathrm{N})$. Estes resultados assemelham-se aos encontrados em experimentos com arroz (Orysa sativa), trigo (Triticum aestivum) e milho (Zea mays), em que, à medida que aumentam os níveis de $\mathrm{N}$ aplicados, ocorre uma diminuição da reflectância em todos os comprimentos de onda, na porção visível do espectro (Patel et al.,1985; Fernández et al., 1994; Blackmer et al., 1996; Ma et al., 1996).

Houve uma diminuição nos valores da reflectância na medida em que aumentou a matéria seca total, a matéria seca verde e o índice de área foliar (Figura 2). Com o aumento da biomassa ocorreu uma diminuição da reflectância nesta faixa do espectro, em virtude da maior absorção da radiação solar incidente na vegetação.

Tabela 1. Valores de índice de área foliar, matéria seca verde, matéria seca total e da reflectância média nas bandas do visível e do infravermelho obtidos em Paspalum notatum nas diferentes datas de medição nos tratamentos ${ }^{(1)}$.

\begin{tabular}{cccccc}
\hline $\begin{array}{c}\text { Dias após início } \\
\text { do experimento }\end{array}$ & $\begin{array}{c}\text { Índice de } \\
\text { área foliar }\end{array}$ & $\begin{array}{c}\text { Matéria seca } \\
\text { verde }\left(\mathrm{g} \mathrm{m}^{-2}\right)\end{array}$ & $\begin{array}{c}\text { Matéria seca } \\
\text { total }\left(\mathrm{g} \mathrm{m}^{-2}\right)\end{array}$ & $\begin{array}{c}\text { Reflectância média } \\
\text { na banda do visivel }\end{array}$ & $\begin{array}{c}\text { Reflectância média na } \\
\text { banda do infravermelho }\end{array}$ \\
\hline 11 & $1,3 \mathrm{ab}$ & $43,8 \mathrm{~b}$ & $\begin{array}{c}0 \mathrm{~kg} \mathrm{~h}^{-1} \mathrm{de} \mathrm{N} \\
108,9 \mathrm{bc}\end{array}$ & $0,081 \mathrm{a}$ & $0,426 \mathrm{a}$ \\
33 & $1,4 \mathrm{ab}$ & $45,6 \mathrm{ab}$ & $115,4 \mathrm{~b}$ & $0,089 \mathrm{a}$ & $0,463 \mathrm{a}$ \\
44 & $1,8 \mathrm{a}$ & $62,3 \mathrm{a}$ & $187,5 \mathrm{a}$ & $0,054 \mathrm{a}$ & $0,431 \mathrm{a}$ \\
60 & $1,0 \mathrm{~b}$ & $34,9 \mathrm{bc}$ & $135,3 \mathrm{~b}$ & $0,121 \mathrm{a}$ & $0,375 \mathrm{a}$ \\
66 & $1,3 \mathrm{ab}$ & $42,2 \mathrm{bc}$ & $134,3 \mathrm{~b}$ & $0,064 \mathrm{a}$ & $0,356 \mathrm{a}$ \\
87 & $0,7 \mathrm{c}$ & $23,0 \mathrm{c}$ & $70,6 \mathrm{c}$ & $0,079 \mathrm{a}$ & $0,304 \mathrm{a}$ \\
\hline & & & $200 \mathrm{~kg} \mathrm{~h}^{-1} \mathrm{de} \mathrm{N}$ & & \\
11 & $2,5 \mathrm{~b}$ & $84,3 \mathrm{~b}$ & $157,0 \mathrm{~b}$ & $0,039 \mathrm{ab}$ & $0,441 \mathrm{a}$ \\
33 & $5,3 \mathrm{a}$ & $177,2 \mathrm{a}$ & $360,9 \mathrm{a}$ & $0,021 \mathrm{~b}$ & $0,377 \mathrm{a}$ \\
44 & $5,6 \mathrm{a}$ & $186,0 \mathrm{a}$ & $363,5 \mathrm{a}$ & $0,028 \mathrm{~b}$ & $0,426 \mathrm{a}$ \\
60 & $1,1 \mathrm{~d}$ & $37,0 \mathrm{~d}$ & $219,2 \mathrm{~b}$ & $0,077 \mathrm{a}$ & $0,296 \mathrm{a}$ \\
66 & $2,3 \mathrm{bc}$ & $76,6 \mathrm{bc}$ & $224,2 \mathrm{~b}$ & $0,062 \mathrm{a}$ & $0,299 \mathrm{a}$ \\
87 & $1,3 \mathrm{~cd}$ & $43,1 \mathrm{~cd}$ & $152,9 \mathrm{~b}$ & $0,067 \mathrm{a}$ & $0,283 \mathrm{a}$ \\
\hline & & & $400 \mathrm{~kg} \mathrm{ha}^{-1} \mathrm{de} \mathrm{N}$ & & \\
11 & $2,6 \mathrm{~b}$ & $85,7 \mathrm{~b}$ & $172,5 \mathrm{c}$ & $0,046 \mathrm{ab}$ & $0,454 \mathrm{a}$ \\
33 & $7,6 \mathrm{a}$ & $254,9 \mathrm{a}$ & $433,5 \mathrm{ab}$ & $0,022 \mathrm{c}$ & $0,394 \mathrm{ab}$ \\
44 & $7,9 \mathrm{a}$ & $263,8 \mathrm{a}$ & $541,2 \mathrm{a}$ & $0,022 \mathrm{c}$ & $0,457 \mathrm{a}$ \\
60 & $1,5 \mathrm{~b}$ & $50,2 \mathrm{~b}$ & $268,9 \mathrm{bc}$ & $0,060 \mathrm{a}$ & $0,235 \mathrm{c}$ \\
66 & $3,8 \mathrm{~b}$ & $127,7 \mathrm{~b}$ & $354,7 \mathrm{bc}$ & $0,034 \mathrm{bc}$ & $0,325 \mathrm{bc}$ \\
87 & $2,4 \mathrm{~b}$ & $80,5 \mathrm{~b}$ & $216,2 \mathrm{c}$ & $0,055 \mathrm{a}$ & $0,273 \mathrm{bc}$ \\
\hline
\end{tabular}

(1)Em relação a cada dose de N, médias seguidas pela mesma letra não diferem entre si pelo teste de Tukey, a 5\% de probabilidade 
Esperava-se que com o aumento da matéria seca total, da matéria seca verde e do índice de área foliar houvesse um correspondente aumento da reflectância média na porção do infravermelho (entre $700 \mathrm{e}$ $950 \mathrm{~nm}$ ). Entretanto, isto não foi observado (Figu- ra 3), provavelmente, devido à grande amplitude de variação dos valores da reflectância (Figura 1), o que pode resultar em uma subestimação da reflectância média, principalmente nos níveis mais baixos de matéria seca (total e verde) e de índice de área foliar.

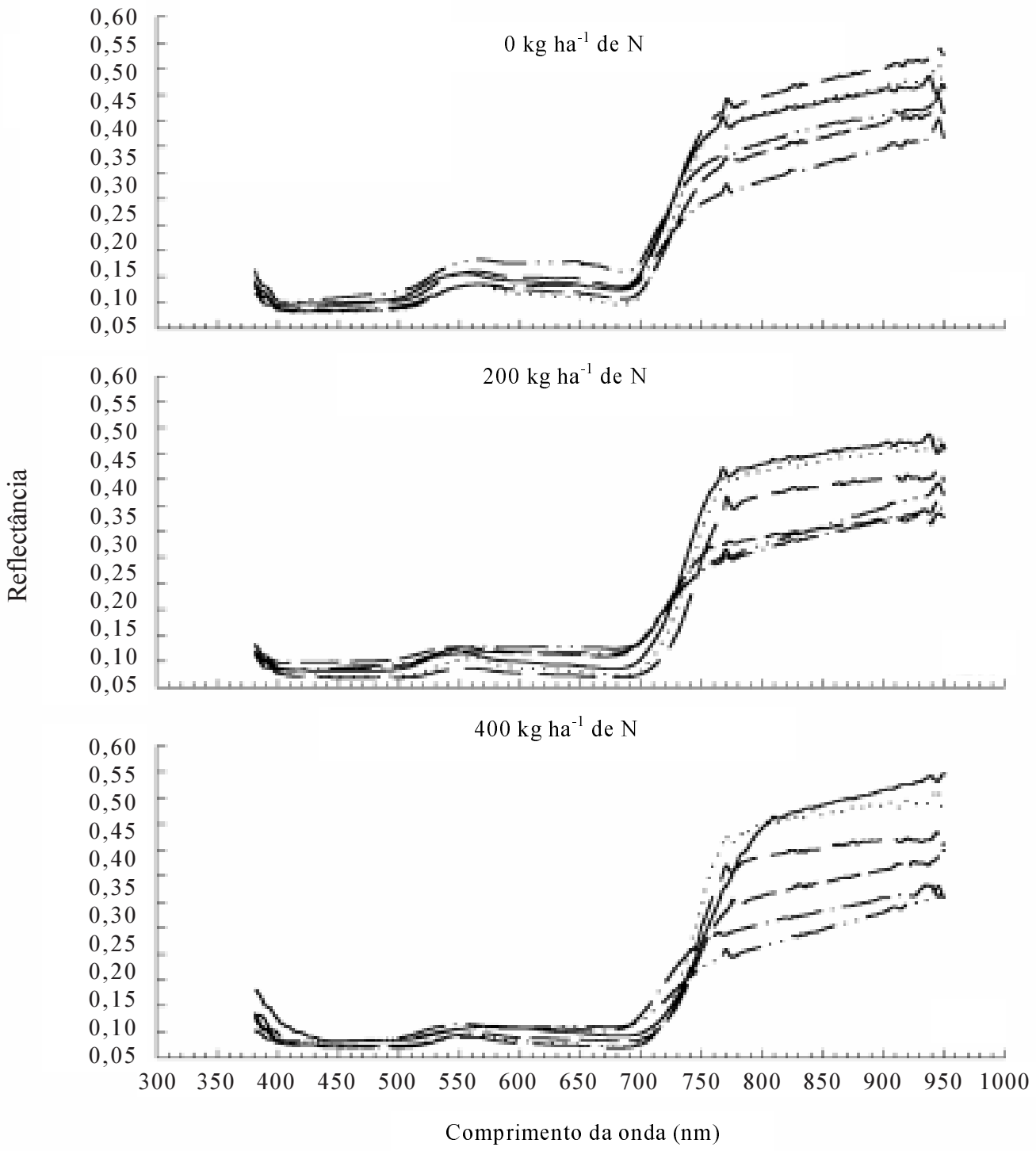

Figura 1. Caracterização espectral de Paspalum notatum aos 11 (- ), 33 (-- ), 44 (...... ), $60(-.-), 66$ (---) e 87 (-- ) dias após o início do experimento nos tratamentos 0, 200 e $400 \mathrm{~kg} \mathrm{ha}^{-1}$ de nitrogênio. EEA/UFRGS, Eldorado do Sul, 1998/1999. 


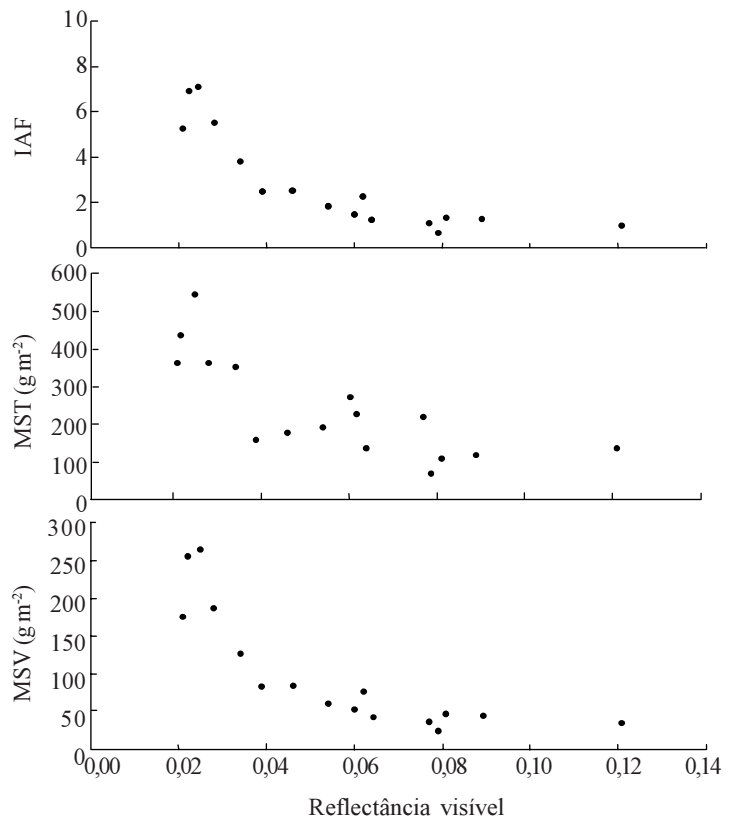

Figura 2. Relação entre a reflectância média da porção visível do espectro e o índice de área foliar (IAF), a matéria seca total (MST) e a matéria seca verde (MSV) em Paspalum notatum. EEA/UFRGS, Eldorado do Sul, 1998/1999.

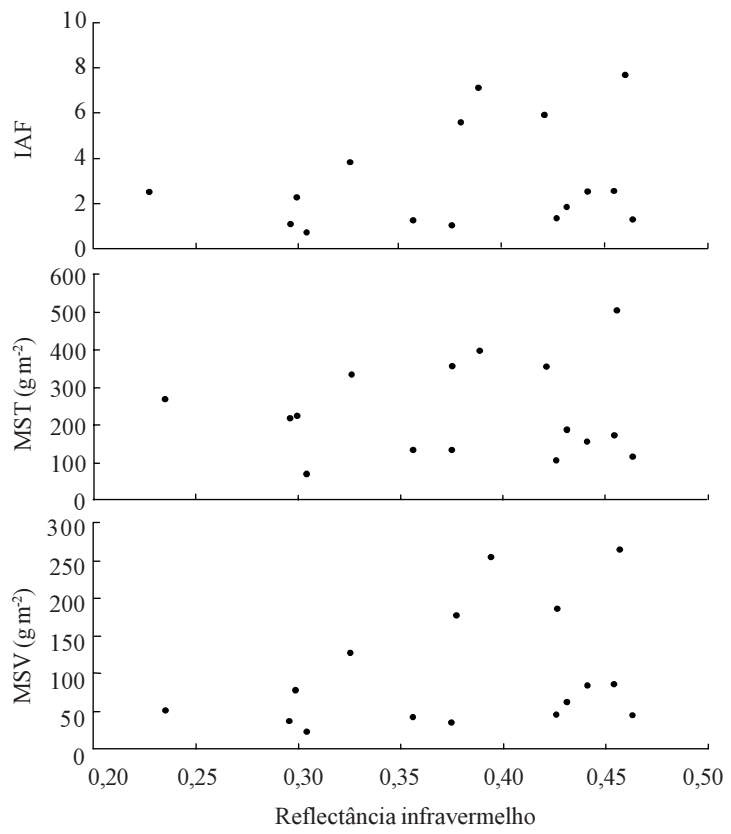

Figura 3. Relação entre a reflectância média na porção do infravermelho do espectro e o índice de área foliar (IAF), a matéria seca total (MST) e a matéria seca verde (MSV) em Paspalum notatum. EEA/UFRGS, Eldorado do Sul, 1998/1999.

\section{Conclusões}

1. A resposta espectral do Paspalum notatum segue os padrões típicos da vegetação verde, e é alterada conforme as variações na quantidade de biomassa do dossel, causadas pelas doses de nitrogênio.

2. Aumentos na quantidade de biomassa diminuem a reflectância na porção visível do espectro e aumentam a reflectância na porção infravermelho.

3. A análise da reflectância é um bom indicador das mudanças que ocorrem em um dossel.

\section{Agradecimentos}

Ao Centro Estadual de Pesquisa em Sensoriamento Remoto e Meteorologia (CEPSRM), pelo empréstimo do espectrorradiômetro; a Capes, pela bolsa concedida à Eliana Lima da Fonseca.

\section{Referências}

BERGAMASCHI, H.; GUADAGNIN, M. R. Agroclima da estação experimental agronômica da UFRGS. Porto Alegre: UFRGS, 1990. $96 \mathrm{p}$.

BLACKMER, T. M.; SCHEPERS, J. S.; VARVEL, G. E; WALTER-SHEA, E. A. Nitrogen deficiency detection using reflected short-wave radiation from irrigated corn canopies. Agronomy Journal, Madison, v. 88, p. 1-5, 1996.

CURRAN, P. J.; MILTON, E. J. The relationships between the chlorophyll concentration, LAI and reflectance of a simple vegetation canopy. International Journal of Remote Sensing, London, v. 4, n. 2, p. 247-255, 1983.

EMBRAPA. Centro Nacional de Pesquisa de Solos (Rio de janeiro, RJ). Sistema brasileiro de classificação de solos. Brasília: Embrapa-SPI, 1999. 412 p.

FERNÁNDEZ, S.; VIDAL, D.; SIMÓN, E.; SOLÉSUGRAÑES, L. Radiometric characteristics of Triticum aestivum cv. Astral under water and nitrogen stress. International Journal of Remote Sensing, London, v. 15, n. 9, p. 1867-1884, 1994.

FIDELES FILHO, J.; BELTRÃO, N. E. M.; RAMANA RAO, T. V. Reflectância do solo seco e úmido e da cultura de amendoim nas bandas do visível e infravermelho próximo. In: CONGRESSO BRASILEIRO DE AGROMETEOROLOGIA 11.; REUNIÃO LATINO-AMERICANA DE AGROMETEOROLOGIA 2., 1999, Florianópolis. 
Anais... Florianópolis: Sociedade Brasileira de Agrometeorologia, 1999. p. 1782-1787.

FONTANA, D. C. Índice de vegetação global para o monitoramento da vegetação e sua correlação com elementos agrometeorológicos e rendimento de grãos de soja. Porto Alegre: UFRGS, 1995. 133 p. Tese de Doutorado.

HALL, D. O.; RAO, K. K. Photosynthesis. Cambridge: Cambridge University Press, 1994. 211 p.

JENSEN, A.; LORENZEN, B.; ØSTERGAARD, H. S.; HVELPLUND, E. K. Radiometric estimation of biomass and nitrogen content of barley grown at different nitrogen levels. International Journal of Remote Sensing, London, v. 11, n. 10, p. 1809-1820, 1990.

LAW, B. E.; WARING, R. H. Remote sensing of leaf area index and radiation intercepted by under story vegetation. Ecological Applications, Washington, v. 4, n. 2, p. 272279, 1994

MA, B. L.; MORRISON, M. J.; DWYER, L. M. Canopy light reflectance and field greenness to assess nitrogen fertilization and yield of maize. Agronomy Journal, Madison, v. 88, p. 915-920, 1996.

MARSCHNER, H. Mineral nutrition of higher plants. London: Academic Press, 1990. 674 p.
PATEL, N. K.; SINGH, T. P.; SAHAI, B.; PATEL, M. S. Spectral response of rice crop and its relation to yield and yield attributes. International Journal of Remote Sensing, London, v. 6, n. 5, p. 657-664, 1985.

PEÑUELAS, J.; ISLA, R.; FILELLA, I.; ARAUS, J. L. Visible and near-infrared reflectance assessment of salinity effects on barley. Crop Science, Madison, v. 37, p. 198202, 1997.

SAS INSTITUTE (Cary, Estados Unidos). SAS user's guide. Cary, 1988. $1028 \mathrm{p}$.

SCURLOCK, H. R.; PRINCE, S. D. Remote sensing of biomass and productivity. In: HALL, D. O.; SCURLOCK, J. M. O.; BOLHÀR-NORDENKAMPF, H. R.; LEEGOOD, R. C.; LONG, S. B. (Ed.). Photosynthesis and production in a changing environment. London: Chapman \& Hall, 1993. p. 22-35.

STEFFEN, C. A.; MORAES, E. C. Tutorial de radiometria. In: SIMPÓSIO BRASILEIRO DE SENSORIAMENTO REMOTO, 7., 1993, Curitiba. Anais... Curitiba: Sociedade Latino-Americana de Sensoriamento Remoto/INPE, 1993. p. 2-12.

WALTER-SHEA, E. A.; NORMAN, J. M.; BLAD, B. L.; ROBINSON, B. F. Leaf reflectance and transmittance in soybean and corn. Agronomy Journal, Madison, v. 83, p. 631-636, 1991. 